visit, 7 were culture positive. MIC profiles were obtained from all isolates.

Conclusion Gonorrhoea was isolated in most asymptomatic men screening positive for $N$. gonorrhoeae by urine NAAT. Clinicians should consider culture in such men to ensure optimal surveillance for antimicrobial resistance. Isolation of $N$. gonorrhoeae in men without discharge indicates these are true infections with viable organisms.

Disclosure No significant relationships.

\section{P625 TRENDS IN SYMPTOMATIC PRESENTATION AMONG REPORTED GONORRHEA CASES, STD SURVEILLANCE NETWORK (SSUN), 2010-2017}

\begin{abstract}
${ }^{1}$ Mark Stenger*, ${ }^{2}$ Emily Weston, ${ }^{3}$ Rachel Amiya, ${ }^{4}$ Emily Han, ${ }^{5}$ Roxanne Kerani, ${ }^{6}$ Ellen Klingler, ${ }^{7}$ Robert Kohn, ${ }^{7}$ Trang Nguyen, ${ }^{8}$ Christina Schumacher, ${ }^{9}$ Elizabeth Torrone. ${ }^{1}$ U.S. Centers for Disease Control and Prevention, Division of STD Prevention, Atlanta, USA ${ }^{2}$ US Centers for Disease Control and Prevention, Division of Sexually Transmitted Diseases, Atlanta, USA; ${ }^{3}$ Washington State Department of Health, Infectious Disease Assessment Unit, Olympia, USA; ${ }^{4}$ California Department of Public Health, STD Control Bureau, Richmond, USA; ${ }^{5}$ Public Health - Seattle and King County, HIVISTD Program, Seattle, USA; ${ }^{6}$ New York City Department of Health and Mental Hygiene, New York City, USA; ${ }^{7}$ San Francisco Department of Public Health, Arches Branch, Population Health Division, San Francisco, USA; ${ }^{8}$ Baltimore City Health Department and Johns Hopkins School of Medicine, Baltimore, USA; ${ }^{9}$ US Centers for Disease Control and Prevention, Division of STD Prevention, Atlanta, USA
\end{abstract}

\subsection{6/sextrans-2019-sti.693}

Background Rates of reported gonorrhea cases have increased in recent years among all groups in the U.S. Expanded screening, particularly extragenital screening among men who have sex with men (MSM), results in increased case finding, complicating interpretation of reported case rates. Monitoring trends in symptomatic presentation through enhanced surveillance may provide insight into changes in case rates.

Methods Randomly sampled cases from 54 counties in 5 U.S. states 2010-2017 were interviewed; symptom status (patient report of 'any STD symptoms') and sex-of-sex partner(s) were elicited. Interviewed cases were weighted to be representative of all reported cases in participating jurisdictions. Proportion of cases presenting with symptoms and symptomatic rate per 100,000 were calculated, stratified by gender and sex-of-sex partners. Trends over time were evaluated by Cochran-Armitage and Pearson's trend tests.

Results During 2010-2017, 21,006 cases were interviewed, representing over 500,000 reported cases. Symptom status was available for 97.1\%; the estimated number of symptomatic cases increased $125.9 \%$ (30,883 to 69,772$)$. The proportion of women and non-MSM males reporting symptoms increased by $15.3 \%(48.3 \%$ to $55.7 \%)$ and $11.5 \%$ (80.1\% to $89.3 \%)$, respectively. Conversely, the proportion of MSM cases reporting symptoms decreased by $23.2 \%(72.5 \%$ to $55.7 \%)$. Relative increase in estimated symptomatic case rate from 2010 to 2017 was $82.8 \%$ among women $(56.0$ to 102.4 per 100,000$)$ , $82.8 \%$ among non-MSM males $(67.0$ to 122.5 per 100,000$)$ and $185.9 \%$ among MSM (946.2 to 2705.2 per 100,000$)$. All trends were significant at $\mathrm{p}<0.05$.
Conclusion Decreases in the proportion of MSM cases reporting symptoms suggests increased case rates among MSM are partially attributable to expanded screening. Yet a significant, increasing trend in the symptomatic case rate among MSM suggests a real increase in gonorrhea transmission. The proportion symptomatic and symptomatic case rates for nonMSM males and females are also rising, suggesting more transmissions in these groups as well.

Disclosure No significant relationships.

\section{P626 NEISSERIA GONORRHOEAE AS AN UNRECOGNIZED CAUSE OF PRESEPTAL CELLULITIS}

${ }^{1}$ Paul Adamson*, ${ }^{2}$ Seth Judson, 'Theo Kelesidis, 'Jeffrey Klausner. 'UCLA - David Geffen School of Medicine, Infectious Diseases, LOs Angeles, USA; ${ }^{2}$ UCLA - David Geffen School of Medicine, Los Angeles, USA

\subsection{6/sextrans-2019-sti.694}

Background Preseptal cellulitis is an infection of the anterior portion of the eyelid and can present with chemosis and eye pain. Preseptal cellulitis and conjunctivitis is a rare, sight threatening infection, is less common in adults, and is usually caused by Streptococcus or Staphylococcus species. Neisseria gonorrhea rarely causes preseptal cellulitis with only four cases previously described.

Methods We describe a case of preseptal cellulitis caused by Neisseria gonorrhea.

Results: Case A 43-year-old woman presented with progressive pain and swelling of her left eye, a low-grade fever and dysuria. On exam, she was afebrile, had significant mucopurulent discharge, eyelid erythema, and a normal cornea. A CT scan of the orbit showed left periorbital preseptal soft tissue swelling. A nucleic acid amplification test (NAAT) for Neisseria gonorrhea was positive in both the left eye swab and a urine specimen. Bacterial cultures from swabs from the eye were also positive for Neisseria gonorrhea. She initially received intravenous (IV) cefepime, vancomycin, piperacillin/tazobactam that were subsequently changed to ceftriaxone, daptomycin and one dose of oral azithromycin. She completed a course of IV ceftriaxone daily for 4 days and 6 additional days of oral cefixime, tobramycin eye drops, and trimethoprim-sulfamethoxazole. She had significant clinical improvement within 3 days and her eye healed well.

Conclusion Clinicians must consider N. gonorrhea infection in patients presenting with acute, unilateral, mucopurulent conjunctivitis and preseptal cellulitis. Rapid diagnosis is critical and we demonstrate the utility of NAAT on an eye specimen. Contact isolation precautions are encouraged, as there is evidence of transmission through fomites and contaminated hands. Treatment data are limited, but we recommend at least 3 days of IV ceftriaxone in combination with azithromycin before transitioning to oral antibiotics based on susceptibilities. In the era of antibiotic resistant gonorrhea, clinicians must be vigilant to ensure appropriate antibiotic treatment of this severe eye infection.

Disclosure No significant relationships. 